\title{
Management of Complicated Crown-root Fracture
}

\author{
${ }^{1}$ Sudheer Kumar Prabhu, ${ }^{2} \mathrm{CU}$ Vivek Chand, ${ }^{3}$ VG Sam Joseph, ${ }^{4}$ Mini K John
}

\begin{abstract}
Traumatic injuries of the teeth and their structures are complex and require comprehensive examination, accurate diagnosis, consideration of various factors involved and a multidisciplinary approach for successful treatment outcome. Dental trauma, in which the fracture line originates in the crown portion of the tooth, extends apically into the root in an oblique direction is referred to as a crown-root fracture. Subgingival fracture of a tooth presents a challenging restorative problem and needs efficient assessment for treatment. A fractured tooth presents a multifaceted problem which warrants a multidisciplinary treatment. A fractured tooth presents a multifaceted problem which warrants a multidisciplinary treatment. Interdisciplinary treatment approach holds true especially in multifaceted problems like traumatic tooth fracture. Every dental professional must be prepared to assess and treat efficiently.
\end{abstract}

Keywords: Computed crown-root fracture, Dental traumatic injuries, Orthodontic extrusion, Subgingival fracture management.

How to cite this article: Prabhu SK, Chand CUV, Joseph VGS, John MK. Management of Complicated Crown-root Fracture. Cons Dent Endod J 2016;1(1):9-13.

Source of support: Nil

Conflict of interest: None

\section{INTRODUCTION}

Dental trauma, in which the fracture line originates in the crown portion of the tooth, extends apically into the root in an oblique direction is referred to as a crown-root fracture. Depending on whether or not the pulp is involved, such fractures are further classified as complicated or uncomplicated. About 5\% of all dental traumas is found to be associated with crown-root fractures. ${ }^{1}$ Severe pain arising from crown-root fractures can be either due to pulpal exposure or due to concomitant periodontal injury or both. Traumatic injuries of the teeth and their structures are complex and require comprehensive examination,

\footnotetext{
1,2Postgraduate Student, ${ }^{3}$ Professor and Head

${ }^{4}$ Assistant Professor

${ }^{1-4}$ Department of Conservative Dentistry and Endodontics Government Dental College, Thiruvananthapuram, Kerala, India

Corresponding Author: CU Vivek Chand, Postgraduate Student, Department of Conservative Dentistry and Endodontics, Government Dental College, Thiruvananthapuram Kerala, India, Phone: 9496468649, e-mail: vivekchandcu@ gmail.com
}

accurate diagnosis, consideration of various factors involved and a multidisciplinary approach for successful treatment outcome. Extension of fracture subgingivally raises concern about biological width violation.

The present paper reports a case of complicated crown fracture in a young adult. The multidisciplinary treatment approach included endodontic treatment of the involved teeth followed by orthodontic extrusion with certain improvisations, periodontal surgery for restoring the biologic width and attaining proper esthetics and placement of definitive crowns.

\section{CASE REPORT}

A 23-year-old male patient reported to the Department of Conservative Dentistry and Endodontics with a complaint of pain in broken upper front teeth. He gave a history of trauma due to a road traffic accident 3 days back. The medical and dental history was found to be noncontributory.

On examination, oblique fracture of teeth \#11, \#21 and \#22 involving pulp was noted. The fracture line was visible labially in all the three teeth involved. However, it was subgingival palatally and mesially in tooth \#21 and \#22. Periodontal probing on the palatal aspect revealed the fracture line to be subgingival $-3 \mathrm{~mm}$ in \#21 and $1 \mathrm{~mm}$ in \#22 (Figs 1A and B).

Radiographic examination revealed fracture of teeth \#11, \#21 and \#22 with an intact periodontal ligament and no root fractures. Number 11 had a fracture on the distal side at the level of alveolar crest, Number 21 with the fracture on the mesial and distal side $2 \mathrm{~mm}$ below alveolar crest and \#22 where the fracture was on mesial side at alveolar crest level. All these fractures involved the pulp space. Based on these findings a diagnosis of complicated crown fracture of tooth \#11 and \#22 and complicated crown-root fracture of tooth \#21 was arrived at Figures 1C and D.

A treatment plan with a sequential, multidisciplinary approach customized for this particular patient was formulated accordingly. The treatment plan was explained in detail to the patient and informed consent was obtained. After routine oral prophylaxis, endodontic treatment of teeth \#11,\#21 and \#22 was carried out (Figs 1E to $\mathrm{H})$. This was followed by post-space preparation and placement of a J-shaped wire which was cemented with zinc phosphate cement (Fig. 1I). Zinc phosphate cement was selected for cementation because of its adequate 

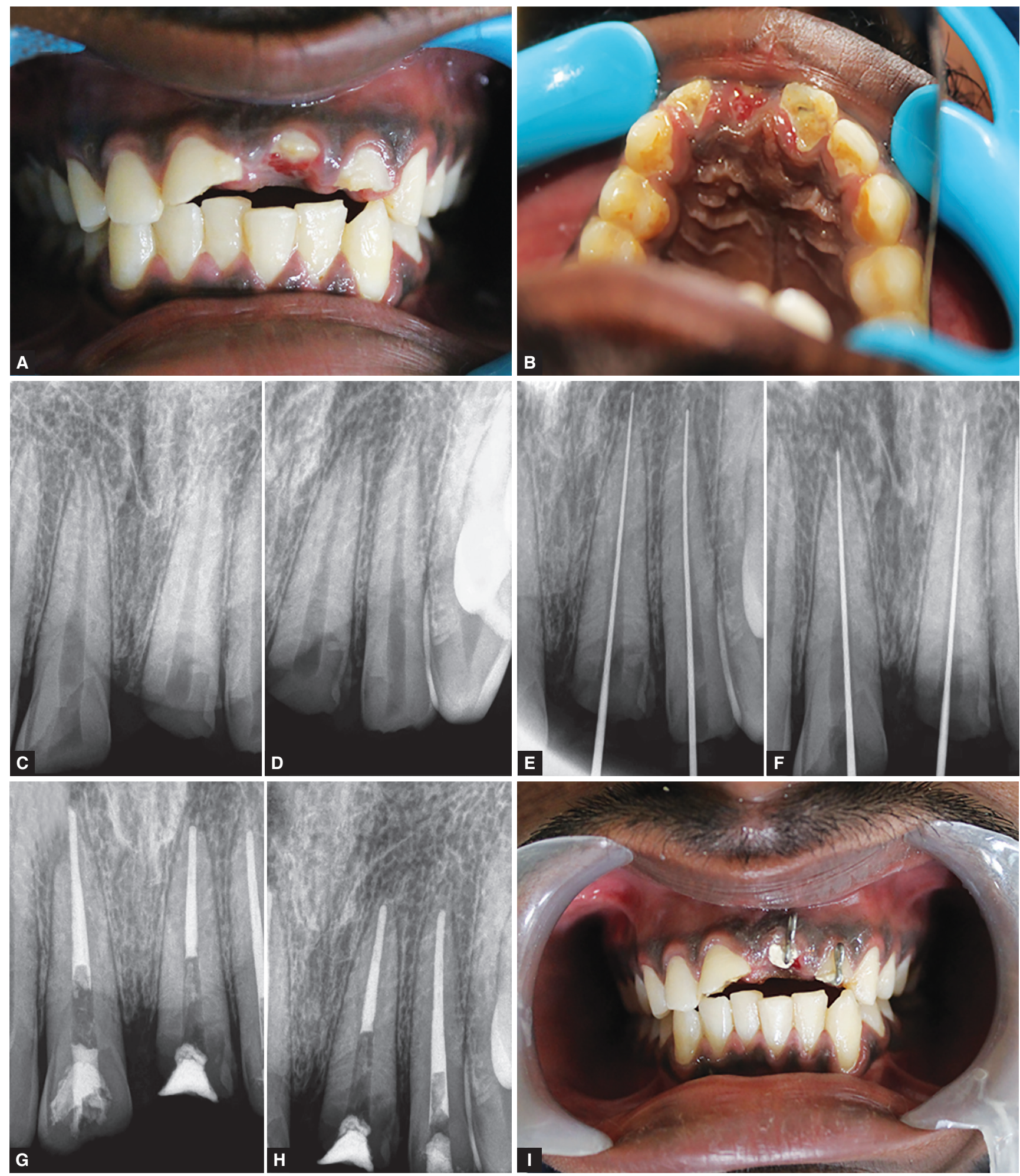

Figs 1A to I: (A) Preoperative photograph (Labial view); (B) Preoperative photograph (Lingual view), (C and D) preoperative radiograph, $(\mathrm{E}$ and $\mathrm{F}$ ) working length determination, $(\mathrm{G}$ and $\mathrm{H}$ ) postobturation radiograph and (I) photograph showing, $\mathrm{J}$ hook cemented to 21 for orthodontic extrusion

strength and ease of removal. Orthodontic brackets were placed on teeth \#13,\#12,\#11,\#23 and \#24. A J hook made of 19 gauge stainless steel wire was luted in teeth \#21 and \#22 and extrusion was effected using $0.16 \mathrm{NiTi}$ wire without interfering with the occlusion. After a period of 2 weeks, $2 \mathrm{~mm}$ of extrusion was achieved. However, in occlusion, the lower incisors were found to be impeding further unhindered extrusion. Since the required crown length had not been achieved, the bite had to be raised by $2 \mathrm{~mm}$ for which an anterior bite plane was employed. This permitted simultaneous crown lengthening and bite opening. Adequate extrusion was achieved in 2 months, 
stabilization was done for 3 months to prevent reintrusion and allow sufficient time for reorganization of the periodontal fibers (Figs 2A and B). The periodontal aspect was addressed with esthetic gingivoplasty in relation to teeth \#12 \#11, \#21, \#22 and \#23, thereby re-establishing the biologic width and proper gingival contour (Figs 2C and D). Finally, the prosthetic treatment was carried out with post-core and full crown on \#21 and full crown on \#11 and \#22. Here a cast post was employed in tooth \#21 since a slightly labial tilt had to be given to achieve sufficient clearance in occlusion (Figs 2E to G).

The patient was then periodically recalled for followup. At 1 year follow-up, clinical evaluation revealed no mobility or pocket formation; but slight gingival inflammation was observed on the buccal aspect of tooth \#21. Radiographic evaluation reveals healthy periodontal and periapical status (Figs 3A to D).

\section{DISCUSSION}

The necessity for an interdisciplinary treatment approach to routine dental problems has been recognized for a long time. This holds true especially in multifaceted problems like traumatic tooth fracture. Traumatic injuries of the teeth and their supporting structures has been and continues to be a challenge, which every dental professional must be prepared to assess and treat efficiently. Extraction should not be the first choice of treatment for such cases, especially in the anterior region; instead, alternative treatment modalities must be considered. Various treatment approaches have been indicated for fractured teeth including: ${ }^{2}$

- Fragment removal followed by restoration ${ }^{5}$

- Fragment reattachment ${ }^{9}$

- Gingivectomy and osteotomy (crown lengthening) ${ }^{9}$

- Orthodontic extrusion with/without gingivoplasty ${ }^{5,9}$
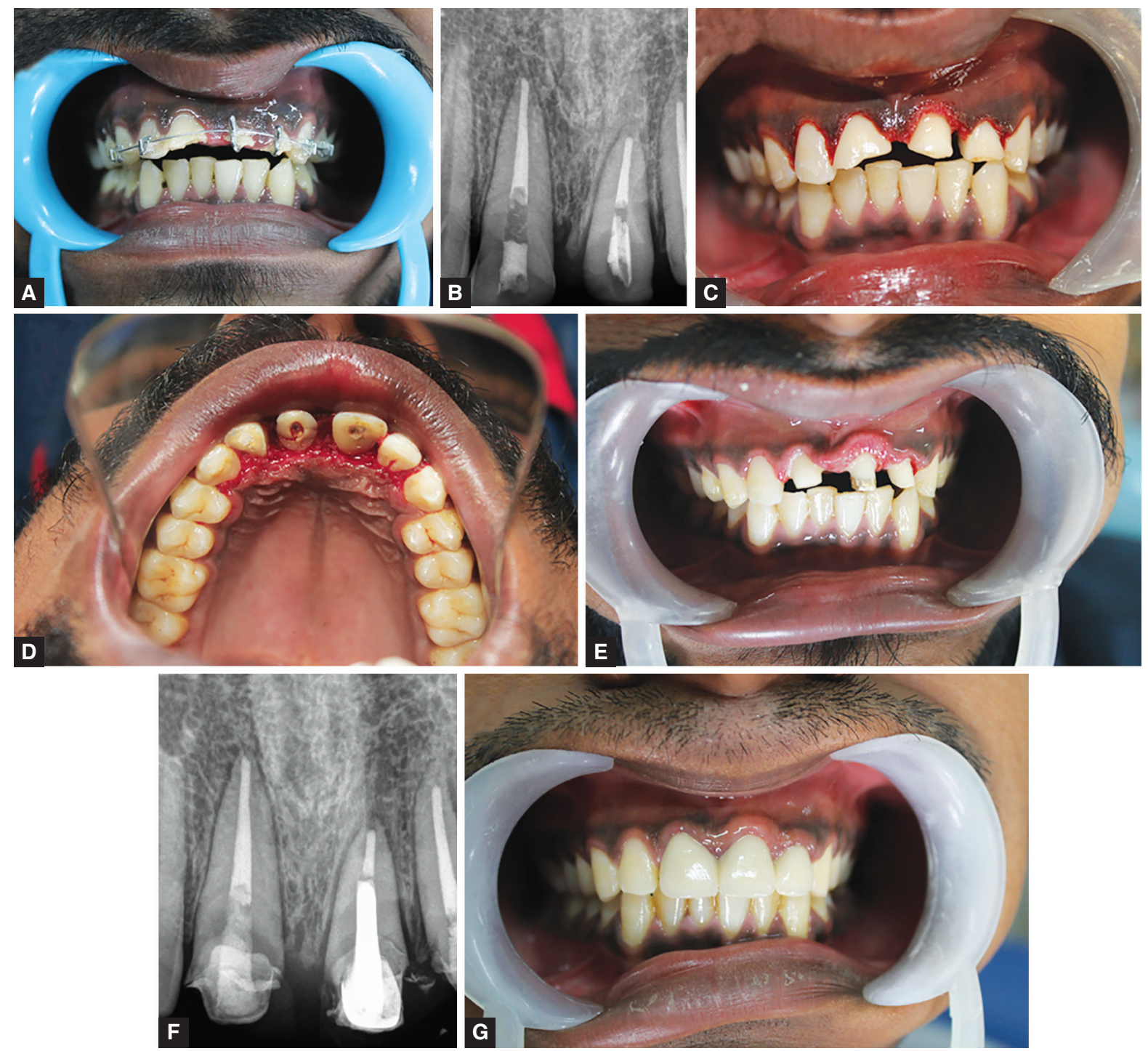

Figs 2A to G: (A) Photograph after orthodontic extrusion, (B) radiograph showing orthodontic extrusion, (C) Photograph after gingivectomy (Labial view), (D) palata view, (E) photograph after cast post cementation in 21, (F) radiograph after case postcementation in 21 (G) photograph after metal ceramic crown cementation in 11, 21, 22 

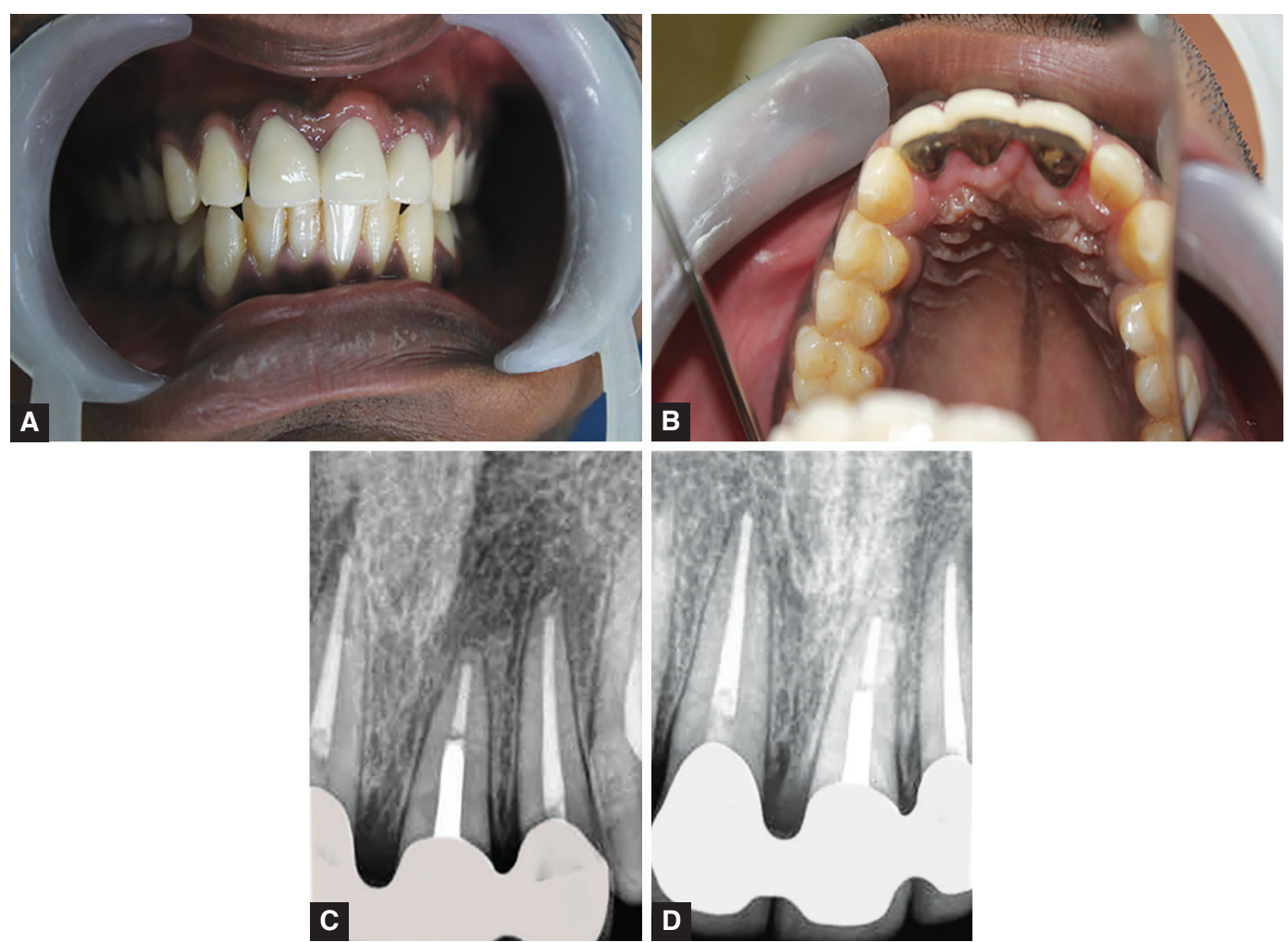

Figs 3A to D: (A) Photograph after 1 year recall (Labial view), (B) palatal view, (C and D) radiograph after 1 year recall

- Forced surgical extrusion 5,9

- Vital root submergence ${ }^{9}$

- Extraction followed by surgical implants ${ }^{9}$ or fixed partial denture. ${ }^{13}$

Subgingival fracture of a tooth presents a challenging restorative problem and needs efficient assessment for treatment. ${ }^{3,4}$ Clinical considerations for the management of crown-root fractures include:

- Extent and pattern of fracture

- Restorability of remaining tooth segment

- Bone support available and its quality

- Damage to the attachment apparatus ${ }^{5,6}$

- Availability of fractured fragment

- Patient cooperation

- Age of the patient

- Esthetics expected by the patient and practically achievable.

In case of fractured anterior teeth preservation of alveolar bone should be a priority, if possible. In the present case location of fractured teeth margin were such that it was difficult to get access to them unless the roots were exposed, either by surgical crown lengthening or orthodontic extrusion.

Controlled orthodontic extrusion is also called as forced eruption, orthodontic eruption, vertical extrusion or assisted eruption and was first reported by Heithersay and Ingber. ${ }^{7-9}$ It can be carried out with removable or fixed orthodontic appliances, the former using mostly elastic bands or magnets and fixed appliances and many modifications. ${ }^{10,11}$ In most cases forced eruption provides a useful alternative to extraction or extensive periodontal surgery, as periodontal crown lengthening can lead to long unesthetic teeth with visible restorative margins. The purpose of this method is not the correction of tooth position in the arch as in conventional orthodontic treatment; it is to preserve the biologic width, which is essential for successful prosthetic rehabilitation. ${ }^{12}$ Additional advantage of forced eruption is that the adjacent teeth need not be prepared for fixed prosthesis and alveolar bone is conserved. Although orthodontic extrusion reduces crown/root ratio and widens the embrasure, this approach allows maintenance of the biologic width and optimizes the marginal sealing of the restoration as it moves the fracture line supragingivally. An important aspect for consideration here is the patient cooperation. Excellent patient cooperation is required for maintenance of proper oral hygiene, use of the orthodontic appliance (if a removable appliance is being used) and periodic visits for reactivation and assessment.

The highlight of this case was the use of an anterior bite plane to enable simultaneous bite opening and tooth extrusion. Continuing with tooth extrusion in spite of hindrance from the opposing arch could result in misdirected forces, change in direction of tooth movement and/or unwanted tooth movement in the opposing arch. A bite plane helps circumvent all these problems while allowing tooth extrusion to go on thus saving time. 


\section{CONCLUSION}

A fractured tooth presents a multifaceted problem which warrants a multidisciplinary treatment. Forced eruption is a very good option to restore the fractured teeth especially in the anterior segment. The advantage of this approach includes preservation of the root structure in order to avoid atrophy of the surrounding bone that normally accompanies a long standing extraction site. The maintenance of the biologic width is also of paramount importance for long-term success of the case. The preservation of bone will enhance the success of eventual implant placement if becomes essential at a later stage.

\section{REFERENCES}

1. Andreason JD, Andreason FM, Andersson L. Textbook and Colour Atlas of Traumatic Injuries to Teeth, Blackwell, Oxford, UK, 4th edition, 2007.

2. Aggarwal V, Logani A, Shah N. Complicated crown fractures-management and treatment options. Int Endodont J 2009 Aug;42(8):740-753.

3. Cortes MI, Marcenes W, Sheiham A. Prevalence and correlates of traumatic injuries to the permanent teeth of schoolchildren aged 9-14 years in Belo Horizonte, Brazil. Dent Traumatol 2001 Feb;17(1):22-26.

4. Marcenes W, Alessi ON, Traebert J. Causes and prevalence of traumatic injuries to the permanent incisors of schoolchildren aged 12 years in Jaragua do Sul, Brazil. Int Dent J 2000 Apr; 50(2):87-92.

5. Olsburgh S, Jacoby T, Krejci I. Crown fractures in the permanent dentition: pulpal and restorative considerations. Dent Traumatol 2002 Jun;18(3):103-115.

6. Reis A, Francci C, Loguercio AD, Carrilho MR, Filho LER. Re-attachment of anterior fractured teeth: fracture strength using different techniques. Operat Dent 2001;26(3):287-294.

7. Oesterle LJ, Wood LW. Raising the root. A look at orthodontic extrusion, JADA 1991;122:193-198.

8. Heithersay GS. Combined endodontic-orthodontic treatment of transverse root fractures in the region of the alveolar crest. Oral Surg Oral Med Oral Pathol 1973 Sep;36(3):404-415.

9. Trushkowsky RD. Esthetic, biologic and restorative considerations in coronal segment reattachment for fractured tooth: a clinical report. J Prosthet Dent 1998;8:139-143.

10. Bondemark L, Kurol J, Hallonsten AL, Andreasen JO. Attractive magnets for orthodontic extrusion of crown-root fractured teeth. Am J Orthod Dentofac Orthop 1997;112: 187-193.

11. Kocadereli I, Tasman F, Guner SB. Combined endodontic orthodontic and prosthodontic treatment of fractured teeth, Case report. Aus Dent J 1998;43:28-31.

12. Stevens B, Levine R. Forced eruption: a multidisciplinary approach for form, function and biologic predictability. Compendium Newtown. 1998;19:994-1012.

13. Meiers JC, Freilich MA. Chairside prefabricated fiberreinforced resin composite fixed partial dentures. Quintessence Int 2001 Feb;32(2):99-104. 